\title{
Efficacy Study of Medial Patellofemoral Ligament Single-bundle Reconstruction with Anterior Half of the Peroneus Longus Tendon Autograft for Treatment of Recurrent Patellar Dislocation
}

Junjun Zha

Jiangsu Taizhou People's Hospital

Tianwei Zhang

Dalian Medical University

E Cai

Dalian Medical University

Ju Tang ( $\square$ tztorchtang@163.com)

Jiangsu Taizhou People's Hospital https://orcid.org/0000-0002-5213-8066

Research article

Keywords: Medial patellofemoral ligament, patellar instability, Reconstruction

Posted Date: August 3rd, 2020

DOI: https://doi.org/10.21203/rs.3.rs-48840/v1

License: (1) (1) This work is licensed under a Creative Commons Attribution 4.0 International License.

Read Full License 


\section{Abstract}

Background To report the clinical outcomes of a longitudinal patellar tunnel technique in reconstruction of the medial patellofemoral ligament(MPFL) with anterior half of the peroneus longus tendon autograft treatment of recurrent patellar dislocation.

Methods From May 2010 to January 2019, we performed MPFL reconstruction with anterior half of the peroneus longus tendon autograft by using a longitudinal patellar tunnel technique on 48 knees in 45 patients(26 female, 19 male) with the median age was 24 (17 44) years old with recurrent patellar dislocation. We made one $4.5 \mathrm{~mm}$ channel from the medial upper edge of the patella to the surface (medial half) of the patella so that to introduce autograft into the medial femoral channel. The autograft was fixed with absorbable screws with the knee bent at $30^{\circ}$. CT and X-rays were used to assess the correction of the tibia tuberosity- trochlear groove (TT-TG) distance, patellar tilt angle, Caton-Deschamps index, femoral anteversion angle, tibial extorsion angle, and the presence or absence of knee valgus. Subjective scores, such as Kujala score and Lysholm score, were used to evaluate knee function preoperatively and postoperatively.

Results No recurrence of patellar dislocation occurred in these patients during an average of $25 \pm$ 7.6 months (range, 6 to 54 months) of follow-up. Preoperative TT-TG distance, patellar tilt angles, and Caton-Deschamps index was $(18.9 \pm 5.7) \mathrm{mm}(10.2 \mathrm{~mm} \sim 32.4 \mathrm{~mm}), 31.5^{\circ} \pm 13.7^{\circ}\left(20.3^{\circ} \sim 58.4^{\circ}\right), 1.1 \pm 0.2$ (1.0 1.5), respectively, which were corrected by $(10.8 \pm 4.3) \mathrm{mm}(4.5 \mathrm{~mm} \sim 17.1 \mathrm{~mm}), 10.7^{\circ} \pm 2.6^{\circ}$ $\left(5.6^{\circ} \sim 15.3^{\circ}\right)$, and $1.07 \pm 0.06(1.02 \sim 1.15)$ postoperatively $(P<0.05) .28$ knees were treated with lateral release + MPFL reconstruction; 11 knees were treated with lateral release + tibial tubercle ingression + MPFL reconstruction; 9 knees were treated with lateral release + tibial tubercle ingression and depression + MPFL reconstruction. At the last follow-up, Lysholm score was $(89.7 \pm 2.3)$, which significantly improved $(P<0.05)$ compared with the pre-operational score of $(54.4 \pm 5.9)$; Kujala score was $(91.5 \pm 4.4)$ points, which significantly improved $(P<0.05)$ compared with the pre-operational score of $(60.6 \pm 5.8)$.

Conclusion One patellar tunnels in reconstruction of the medial patellofemoral ligament (MPFL) with anterior half of the peroneus longus tendon autograft is a safe, effective, and economic method for recurrent patellar dislocation.

\section{Background}

Recurrent patellar dislocation commonly occurs in young people and is often accompanied by one or more anatomical abnormalities, which seriously affect the patient's life and motor function. Thomas et al. ${ }^{[1]}$ confirmed that patellar dislocation is an important risk factor of patellofemoral arthritis. Nearly half of patients develop symptom of arthritis after 25-year lateral patellar dislocation. Surgical procedure is currently the most reliable treatment method ${ }^{[2,3]}$. The medial patellofemoral ligament (MPFL) is the major medial structure to restrain lateral patellar displacement, contributes $50-60 \%$ of the total restraining force 
of medial patellar, so MPFL reconstruction has become an important surgery for treating patellar instability ${ }^{[4-6]}$.

Currently, there are a variety of MPFL reconstruction techniques, such as reconstruction with reconstruction with autograft tendon (including gracilis, semitendinosus, and peroneus longus tendon), ligament reconstruction with allograft or synthetic materials ${ }^{[5]}$. The graft can be fixed by various techniques, including patella drilling, suture, suture anchor, and fixation screw on the femoral condyle. Overall, the clinical result of MPFL reconstruction techniques is successful. The re-dislocation rate reported from literatures is less than $10 \%{ }^{[2,7-9]}$. For techniques involving lateral patella drilling, the incidence of postoperative patella fracture is also low ${ }^{[10]}$.

This study reported the clinical results of 45 patients undergoing MPFL reconstruction for the treatment of patellar instability. Patients receive MPFL single-bundle reconstruction with anterior half of the peroneus longus tendon autograft, screw fixation in femoral condyle, in combination with lateral patellar retinacular release technique or lateral patellar retinacular release or tibial tubercle displacement technique, to evaluate the clinical efficacy of medial patellar ligament single-bundle reconstruction and the incidence of complications such as patellar fracture and re-dislocation.

\section{Methods}

This study included all patients undergoing MRFL reconstruction from May 2010 to January 2019. The average follow-up time was 25 months ( 6 54 months). There were 19 males (20 knees) and 26 females (28 knees). The median age was 24 (17 44) years old. All patients had chronic patellar instability, with patellar dislocation at least twice. Exclusion criteria:(1)Patients with significant degenerative change of patellofemoral joint (grade III-IV);(2)Patients with generalized ligament laxity;Patients with knee valgus and knee hyperextension; (3)femoral anteversion angle $>25^{\circ}$; (4)tibial extorsion angle $>40^{\circ}$. Severe trochlear dysplasia (Dejour type C or type D).

All patients underwent knee joint lateral and lower extremity standing anteroposterior X-ray, lower extremity CT scan and knee joint MRI. Measures include patellar tilt angle, tibial tubercle-trochlear groove distance (TT-TG distance), Caton-Deschamps index, femoral anteversion angle, tibial extorsion angle, and the presence or absence of knee valgus. The knee joint lateral X-ray confirmed the femoral trochlear dysplasia and performed Dejour typing. All patients had Kujala score and Lysholm score.

\section{Determination of combined surgery plan}

Patellar lateral retinacular release technique is performed in patients with patella tilt angle $>20^{\circ}$; tibial tubercle displacement technique is performed in patients with TT-TG distance $>20 \mathrm{~mm}$, to shorten the TTTG distance to be $10 \mathrm{~mm}$ after displacement; tibial tubercle displacement technique is performed in patients with Caton-Deschamps index $>1.2$, to adjust it to about 1.0 after displacement.

\section{Surgical methods}


Lateral patellar retinacular release: It is performed using a plasma knife under arthroscopy. The surgical range extends from the lateral edge of the tibial tubercle, along the lateral edge of the patellar tendon to the inferior patellar, and travels proximally along the anterior border of the iliotibial band with the turn until the lateral femoral aponeurosis-muscle transition.

Tibial tubercle displacement: Make a $4-5 \mathrm{~cm}$ longitudinal incision along the lateral edge of the tibial tubercle, reveal the entire tibial tubercle in subcutaneous separation, and dissect the muscle to reveal the lateral surface of the tibial tubercle. Use a small pendulum saw along the medial margin of the patellar ligament, starting from the patellar ligament tibial tubercle point to cut $5.0 \mathrm{~cm}$ distally. Towards the distal sawing site, cut diagonally and medially from the patellar ligament tibial tubercle point along the lateral edge of the patellar ligament. The two sawing sections intersect at the far end. Finally, saw off at the deep surface of the patellar ligament and at the tibial tubercle point. The length is the width of the patellar ligament, and it intersects with the two previous sawed sections. All three sawing sections have the depth of $1.0 \mathrm{~cm}$. The lateral triangular tibial tubercle osteotomy block is made of the patellar ligament. A medial triangular osteotomy block is taken inside or below the osteotomy block, so that the tibial tubercle osteotomy block with patellar ligament can be moved inward or downward according to the preoperative plan. After the inward or downward movement is completed, the medial osteotomy block is exchanged and filled to the lateral bone defect. Fix the osteotomy block with patellar ligament using screw or Kirschner wire.

The peroneus longus tendon anterior half of the affected side was used as a graft to reconstruct MPFL. The diameter of the graft was 3 to $3.5 \mathrm{~mm}$, and the absorbable threads at both ends were sutured. First, perform arthroscopy to handle intra-articular synovitis, cartilage damage and loose body. Touch the upper and lower patella and the widest part of the patella (generally the junction of the upper third of the patella and the MPFL attachment point) in vitro. Make a $1 \mathrm{~cm}$ longitudinal incision on the inside of the widest part of the patella. Through this incision, use a 2.0 Kirschner wire to drill from the junction between the inner edge of the patella and the vertical articular surface of the patella to the midline of the patella surface, and a $4.5 \mathrm{~mm}$ hollow drill to expand the bone tunnel. On the body surface, touch the femoral medial epicondyle and adductor tubercle, and make a longitudinal incision of about $2 \mathrm{~cm}$ on the surface until the femoral medial epicondyle, adductor tubercle, and gastrocnemius tubercle are revealed. Drill a $6 \mathrm{~mm}$ bone tunnel obliquely near, anteriorly and laterally at the depressed site of the three points of the medial epicondyle of the femur, the adductor tubercle and the gastrocnemius tubercle. The diameter of the tunnel is generally slightly larger than the diameter of the double femoral tendons. Pull one end of the tendon from the channel of the medial edge of the patella, pull out from the anterior channel of the patella, reflex the tendon, and pull the tendon back along the front of the patella. Pull the tendon medially to flex the knee gradually to $90^{\circ}$, and check the inward ROM of patellar, patellar reduction, and lateral patellar tissue tension. Pull both ends of the tendon through the shallow deep fascia, pull out from the incision of the adductor tubercle, and then pull the tendon into the femoral tunnel. Flex the knee at $30^{\circ}$, gently pull the suture at the end of the tendon, push the patella outward to confirm that the patella is not outward instable, and fix with a $7 \mathrm{~mm} \times 25 \mathrm{~mm}$ absorbable compression screw. Before the end of 
operation, reconfirm that the patella is not dislocated, and the joint can be bent freely to $110^{\circ}$ without restriction.

The same rehabilitation procedures were used after surgery, and braces were worn to keep the joints in the extended position within 4 weeks after surgery. Since Day 2 after the operation, the patients started the isometric quadriceps femoris contraction exercises and the straight leg raise exercises. The patients started partial weight-bearing 2 weeks after surgery, and full weight-bearing 4 weeks after surgery. The patients started the knee flexion exercises 5 days after surgery, and the knee flexion to $90^{\circ} 2$ weeks after surgery. The angle of knee flexion gradually increased afterwards, and the knee flexion returned normal after 2 months. The patients resumed jogging 4 months after surgery and normal physical activity 6 months after surgery.

The patients were followed up 3 months, 6 months and 1 year and after surgery, every year afterwards. The frontal and lateral X-rays and CT scan of the affected knee were performed, and Kujala score and Lysholm score were evaluated. The patients with bilateral affected knee joints were evaluated separately on each limb.

\section{Statistical analysis}

Statistical analyses were performed using the SPSS version 19. The patellar tilt angle, TT-TG distance, Caton-Deschamps index, Lysholm score and Kujala score were summarized using mean \pm standard deviation. The patients were divided into two groups of pre-operation group and post-operation group, .and the paired sample t test was conducted between the two groups at the level of $a=0.05$.

\section{Results}

All patients were followed up after the operation. The follow-up time was $6 \sim 54$ months, with an average of $25 \pm 7.6$ months. During the follow-up period, all patients had good knee flexion and extension activities, and experienced no re-dislocation or subluxation, as well as no other obvious complications. At the last follow-up, Lysholm score was $(89.7 \pm 2.3)$, which significantly improved $(P<0.05)$ compared with the pre-operational score of (54.4 \pm 5.9$)$; Kujala score was $(91.5 \pm 4.4)$ points, which significantly improved $(P<0.05)$ compared with the pre-operational score of $(60.6 \pm 5.8)$.

Before the operation, the average patellar tilt angle was $31.5^{\circ} \pm 13.7^{\circ}\left(20.3^{\circ} \sim 58.4^{\circ}\right)$; the average TT-TG distance was $(18.9 \pm 5.7) \mathrm{mm}(10.2 \mathrm{~mm} \sim 32.4 \mathrm{~mm})$; the average Caton-Deschamps index was $1.1 \pm 0.2$ (1.0 1.5). 28 knees were treated with lateral release + MPFL reconstruction; 11 knees were treated with lateral release + tibial tubercle ingression + MPFL reconstruction; 9 knees were treated with lateral release + tibial tubercle ingression and depression + MPFL reconstruction. After the operation, the average patellar tilt angle was $10.7^{\circ} \pm 2.6^{\circ}\left(5.6^{\circ} \sim 15.3^{\circ}\right)(\mathrm{P}<0.05)$; the average TT-TG distance was $(10.8 \pm 4.3) \mathrm{mm}$ $(4.5 \mathrm{~mm} \sim 17.1 \mathrm{~mm})(P<0.05)$; and the average Caton-Deschamps index was $1.07 \pm 0.06(1.02 \sim 1.15)(P$ $>0.05)$. 


\section{Discussion}

The habitual patellar dislocation is often accompanied by multiple anatomical abnormalities and medial retinacular injury of knee joint, and surgical procedure is mostly used clinically $[2,3]$. In patients with acute patellar dislocation, there is a high probability of injury of medial patellofemoral ligament of the knee ${ }^{[4,11}$, 12]. Furthermore, some studies ${ }^{[4,6]}$ confirmed that the medial patellofemoral ligament of the knee can provide $50 \% \sim 60 \%$ of the medial restraint of the patella flexed at $0^{\circ} \sim 30^{\circ}$, and play a major role in maintaining patellar stability. Therefore, MPFL is the leading stable structure of medial patella, and MPFL reconstruction is increasingly focused on ${ }^{[7]}$.

However, it should be noted that MPFL reconstruction can only anatomically restore the static stable structure of medial patella, and cannot correct the abnormality in lower extremity bony lines. Hopper et al $^{[13]}$ found that in their study, among patients undergoing MPFL reconstruction, the relocation rate in patients with severe trochlear dysplasia was $100 \%$, but in patients with low-grade trochlear dysplasia was 9.3\%. Schöttle et al. ${ }^{[8]}$ studied the relationship between arthroscopic MPFL repair and trochlear dysplasia, and found that at 12-month follow-up, 0/26 knees had re-dislocation in the none/mild trochlear dysplasia group, while 4/22 knees had re-dislocation in the severe trochlear dysplasia group. In the severer trochlear dysplasia group, the surgical efficacy was limited, indicating that MPFL reconstruction alone was not enough. Therefore, for patients with concurrent changes in bone structure such as high patella, knee valgus or retroflexion deformity, femoral internal and external rotation, tibial tubercle ingression and severe femoral trochlear dysplasia, osteotomy orthopedics, tibial tubercle ingression surgery and femoral trochlear plasty are often required. Therefore, for patients with recurrent patellar dislocation, the appropriate surgical procedure should be selected based on comprehensive analysis and judgment from detailed physical examinations and comprehensive imaging and arthroscopic findings.

For patients with patellar inclination angle $>20^{\circ}$, MPFL reconstruction in combination with lateral patellar retinacular release technique can be performed to increase the ingression degree of patellar. However, some studies have shown that the lateral retinaculum plays only a secondary role in the patellar soft tissue stable structure ${ }^{[14]}$. Therefore, lateral retinacular release alone may have a good early surgical effect, but the effect will gradually decline over time ${ }^{[15]}$. In this study, the technique was only used as part of the combined surgery to loosen the lateral patellar traction structure and increase the ingression degree of patellar. During the intraoperative retinacular release under microscopy, at $1-1.5 \mathrm{~cm}$ from the outer edge of the patella, use a plasma radiofrequency scalpel to gradually loosen from the proximal end to the distal end to reach deep into the subcutaneous fat layer or muscular layer. Usually after full release, widening of the lateral patellofemoral space or an increase in patellar lateral ROM can be observed. In this study, the patient's patellar tilt angle was corrected from $31.5^{\circ} \pm 13.7^{\circ}\left(20.3^{\circ} \sim 58.4^{\circ}\right)$ before operation to $10.7^{\circ} \pm 2.6^{\circ}\left(5.6^{\circ} \sim 15.3^{\circ}\right)$ after operation $(P<0.05)$, lower than the surgical threshold. The postoperative effect was good. 
Stephen et al. ${ }^{[16]}$ studied the effect of different TT-TG distances on the surgical effect in 8 knee joint specimens. The results showed that in the normal anatomical position of the tibial tubercle and within $5 \mathrm{~mm}$ range of outward displacement, the MPFL reconstruction can correct the patellar outward displacement and restore normal medial patellofemoral joint pressure and patellar tilt angle, so it is recommended that if the TT-TG distance is $>15 \mathrm{~mm}$, tibial tubercle ingression surgery should be considered. Schöttle et al. ${ }^{[8]}$ believed that the clinical effect of MPFL reconstruction alone or lateral retinacular release alone was not good in patients with concurrent femoral trochlear severe dysplasia. In this study, if TT-TG distance was $>20 \mathrm{~mm}$, the tibial tubercle ingression was performed to make the TTTG distance close to $10 \mathrm{~mm}$ after ingression. With regard to the patellar depression limit, it's believed that the patellar depression limit was $10.0 \mathrm{~mm}$, or it may cause excessive pressure on the patellofemoral joints and resultant postoperative knee pain. In this study, 20 knees were treated with tibial tubercle osteotomy, of which 11 knees were treated with lateral release + tibial tubercle ingression + MPFL reconstruction, and 9 knees were treated with lateral release + tibial tubercle ingression and depression + MPFL reconstruction. The average postoperative TT-TG distance improved to $4.5 \mathrm{~mm} \sim 17.1 \mathrm{~mm}(10.8 \pm$ 4.3) $\mathrm{mm}(P<0.05)$. The average Caton-Deschamps index improved to $1.02 \sim 1.15(1.07 \pm 0.06),(P>0.05)$. The clinical efficacy was good.

The initial tension required for MPFL graft fixation should be appropriate, but it is difficult to be determined accurately and there is currently no clear reference standard. In this study, the MPFL reconstruction with $30^{\circ}$ flexion was generally used in the study. The knee joint was fully flexed and extended. At the same time, the patellar trajectory and reduction alignment were observed under arthroscopy to confirm that the patella was well stabilized with no lateral dislocation and patellar lateral tilting $I^{\circ}$, suggesting that the graft tension was appropriate for fixation.

This study had certain limitations, and minors were not included in this study due to involvement of epiphysis. The sample number was small and the follow-up time was short, so there may be errors in the reported results.

\section{Conclusion}

In this study, the patients had ideal postoperative motion function recovery, due to the careful imaging evaluation before surgery, the appropriate selected combined surgical methods of combined lateral retinacular release and tibial tubercle osteotomy as appropriate for treatment of recurrent patellar dislocation. The short-term clinical efficacy was satisfactory. However, the longest follow-up time for patients in this group was 35 months, so a large number of further clinical studies and long-term followup for efficacy observation are still needed.

\section{List Of Abbreviations}

1. medial patellofemoral ligament(MPFL) 
2. tibia tuberosity- trochlear groove (TT-TG)

\section{Declarations}

\section{Trial registration}

Current Controlled Trials ChiCTR2000034347. Registered 3 July 2020.

\section{Ethics approval and consent to participate}

This study has been registered in the ethical committee of our hospital(Taizhou people's hospital, Taizhou, Jiangsu), and all patients gave informed consent(the consent we obtained from study participants was written).

\section{Consent for publication}

All images have been consented for publication.

\section{Availability of data and materials}

The datasets are available from the corresponding author on reasonable request.

\section{Competing interests}

No benefits in any form have been received or will be received from a commercial party related directly or indirectly to the subject of this article.

\section{Funding}

There is no funding in this research.

\section{Authors' contributions}

Tw Z, E C analyzed and interpreted the patient data. J T,Jj Z analyzed the clinical outcomes .All authors have read and approved the final manuscript.

\section{Acknowledgements}




\section{References}

1. Thomas L, Sanders A, Pareek NR, Johnson, et al. Patellofemoral Arthritis After Lateral Patellar Dislocation[J]. Am J Sports Med.

2. Enderlein D, Nielsen T, Christiansen SE, et al. Clinical outcome after reconstruction of the medial patellofemoral ligament in patients with recurrent patella instability[J]. Knee Surg Sports Traumatol Arthrosc. 2014;22(10):2458-64.

3. Fithian DC, Paxton EW, Stone ML, et al. Epidemiology and Natural History of Acute Patellar Dislocation[J]. Am J Sports Med. 2004;32(5):1114-21.

4. Samuel D, Russ M, Tompkins D, Nuckley, et al. Biomechanical Comparison of Patellar Fixation Techniques in Medial Patellofemoral Ligament Reconstruction[J]. Am J Sports Med. 2015;43:195-9.

5. Lee PYF, Golding D, Rozewicz S, et al. Modern synthetic material is a safe and effective alternative for medial patellofemoral ligament reconstruction[J]. Knee Surg Sports Traumatol Arthrosc. 2018;26(9):2716-21.

6. Feller JA, Amis AA, Andrish JT, et al. Surgical Biomechanics of the Patellofemoral Joint[J]. Arthrosc J Arthrosc Relat Surg. 2007;23(5):542-53.

7. Shamrock AG, Day MA, Duchman KR, et al. Medial Patellofemoral Ligament Reconstruction in Skeletally Immature Patients: A Systematic Review and Meta-analysis[J]. Orthop J Sports Med. 2019;7(7):232596711985502.

8. Schöttle PB, Scheffler SU, Schwarck A, et al. Arthroscopic Medial Retinacular Repair After Patellar Dislocation With and Without Underlying Trochlear Dysplasia: A Preliminary Report[J]. Arthrosc J Arthrosc Relat Surg. 2006;22(11):1192-8.

9. Deie $M$, Ochi $M$, Sumen $Y$, et al. A long-term follow-up study after medial patellofemoral ligament reconstruction using the transferred semitendinosus tendon for patellar dislocation[J]. Knee Surg Sports Traumatol Arthrosc. 2005;13(7):522-8.

10. Shah JN, Howard JS, Flanigan DC, et al. A Systematic Review of Complications and Failures Associated With Medial Patellofemoral Ligament Reconstruction for Recurrent Patellar Dislocation[J]. Am J Sports Med. 2012;40(8):1916-23.

11. Arendt EA, Fithian DC, Cohen E. Current concepts of lateral patella dislocation[J]. Clin Sports Med. 2002;21(3):499-519.

12. Sanders TG, Morrison WB, Singleton BA, et al. Medial Patellofemoral Ligament Injury Following Acute Transient Dislocation of the Patella: MR Findings with Surgical Correlation in 14 Patients:[J]. J Comput Assist Tomogr. 2001;25(6):957-62.

13. Hopper GP, Leach WJ, Rooney BP, et al. Does Degree of Trochlear Dysplasia and Position of Femoral Tunnel Influence Outcome After Medial Patellofemoral Ligament Reconstruction?[J]. Am J Sports Med. 2014;42(3):716-22. 
14. Greg Harasen. Patellar luxation: Pathogenesis and surgical correction[J]. Can Vet J. 2006;47(10):1037-9.

15. Dandy DJ, Desai SS. The results of arthroscopic lateral release of the extensor mechanism for recurrent dislocation of the patella after 8 years[J]. Arthrosc J Arthrosc Relat Surg. 1994;10(5):540-5.

16. Stephen M, Desio RT, Burks, Kent N, Bachus. Soft tissue restraints to lateral patellar translation in the human knee[J]. Am J Sports Med. 1998;26(1):59-65.

\section{Figures}




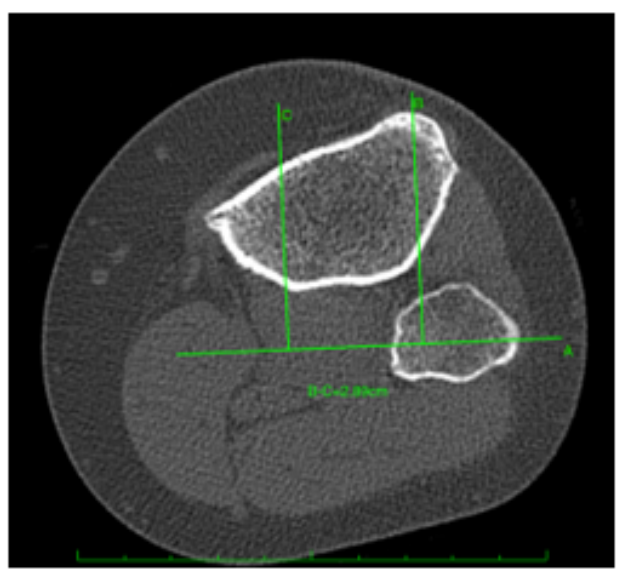

A

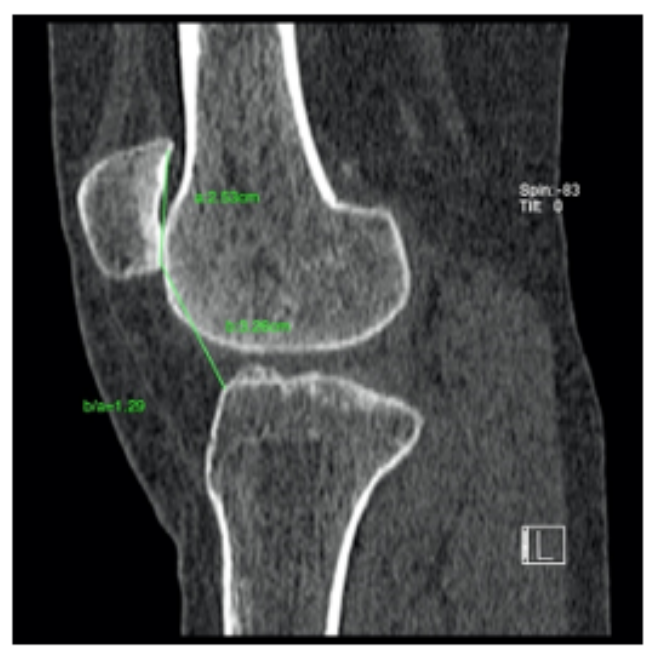

C

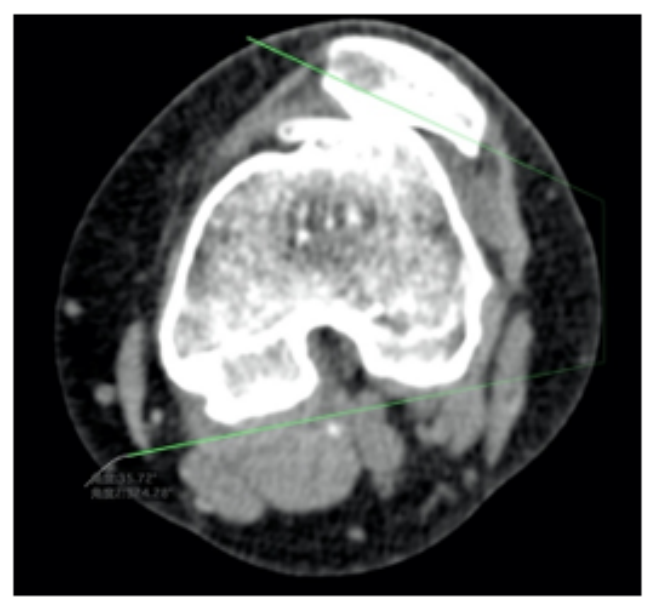

$\mathrm{E}$

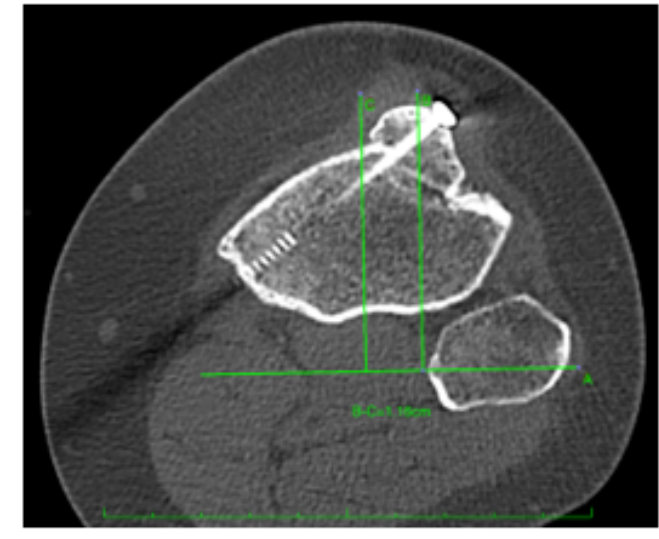

B

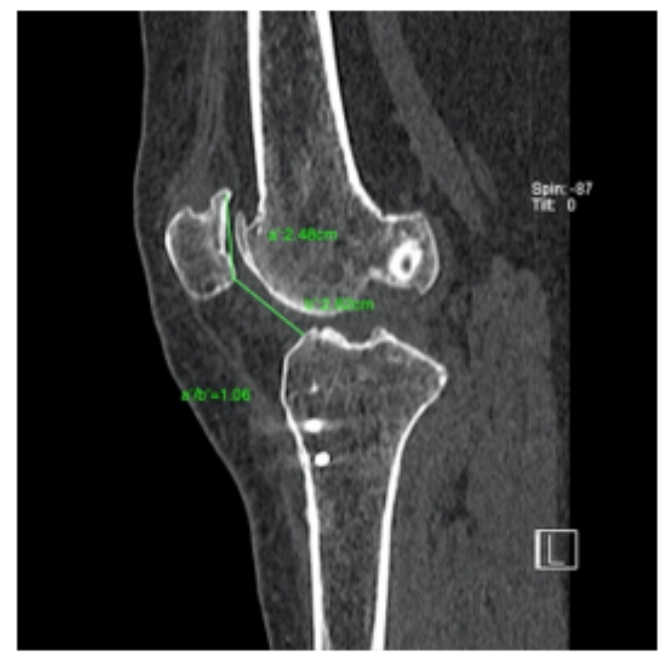

D

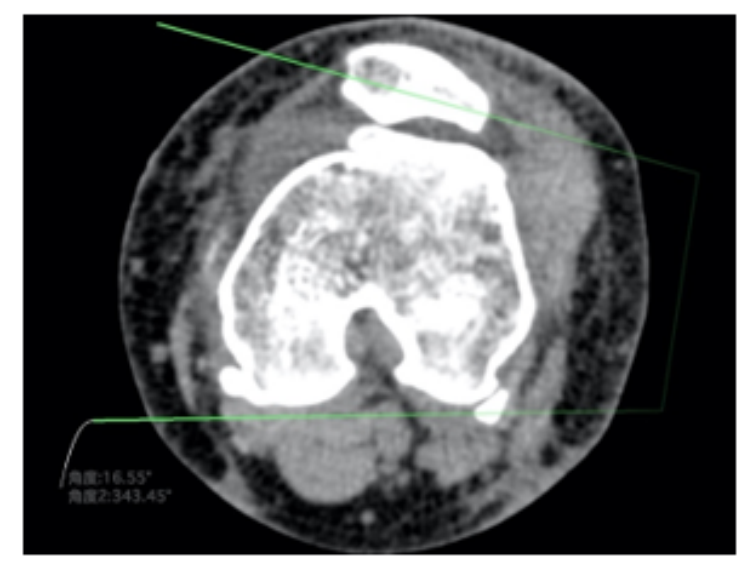

$\mathrm{F}$

\section{Figure 1}

The TT-TG value, Caton-Deschamps index and tilt angle of patella were compared under CT image(22 years old, female, treated with lateral release + tibial tubercle ingression and depression + MPFL reconstruction).A: TT-TG preoperative values: $2.89 \mathrm{~cm}$. B: TT-TG postoperative values: $1.16 \mathrm{~cm}$. C: Preoperative Caton-Deschamps index:1.29. D: Postoperative Caton-Deschamps index:1.06. E: Preoperative patella tilt angle:35.72 . F: Postoperative patella tilt angle: $16.55^{\circ}$. 